\title{
Some comments on the second virial coefficient of semiflexible polymers
}

$\operatorname{AUTHOR}(\mathrm{S})$ :

Ida, Daichi; Yoshizaki, Takenao

CITATION:

Ida, Daichi ...[et al]. Some comments on the second virial coefficient of semiflexible polymers. JOURNAL OF CHEMICAL PHYSICS 2008, 129(16): 164902.

ISSUE DATE:

2008-10-28

URL:

http://hdl.handle.net/2433/84609

\section{RIGHT:}

Copyright 2008 American Institute of Physics. This article may be downloaded for personal use only. Any other use requires prior permission of the author and the American Institute of Physics. 


\title{
Some comments on the second virial coefficient of semiflexible polymers
}

\author{
Daichi Ida and Takenao Yoshizaki ${ }^{\mathrm{a})}$ \\ Department of Polymer Chemistry, Kyoto University, Katsura, Kyoto 615-8510, Japan
}

(Received 28 May 2008; accepted 17 July 2008; published online 24 October 2008)

\begin{abstract}
A Monte Carlo study is made of the mean-square radius of gyration $\left\langle S^{2}\right\rangle$ and second virial coefficient $A_{2}$ for the two freely rotating chains with the Lennard-Jones (LJ) 6-12 potential and the hard-sphere (HS) one in the range of the bond angle $\theta$ from $109^{\circ}$ (typical flexible chain) to $175^{\circ}$ (typical semiflexible or stiff chain) and in the range of the number $n$ of bonds from 6 to 1000 . It is shown that a value may be properly assigned to the collision diameter of the HS potential so that $\left\langle S^{2}\right\rangle$ of the chain with the HS potential agrees well with that of the chain with the LJ one whose parameter values correspond to a good-solvent condition irrespective of the chain stiffness. It is then found that $A_{2}$ of the latter chain becomes remarkably smaller than that of the former as the chain stiffness is increased. The result implies that the binary-cluster approximation does not seem to work well for typical semiflexible and stiff polymers. (C) 2008 American Institute of Physics. [DOI: 10.1063/1.2969106]
\end{abstract}

\section{INTRODUCTION}

When we consider dilute solution behavior of nonionic flexible polymers in good solvents, interactions between segments constituting the polymer chains are all, without exception, treated in the binary-cluster approximation in the frameworks of both the (conventional) perturbation theory ${ }^{1}$ and the polymer renormalization group theory. ${ }^{2-4}$ The approximation assumes that the effect of the interactions, i.e., the excludedvolume effect, does not depend on a detailed form of the pair potential $u(R)$ of mean force between the segments as a function of their separation $R$ but only on the binary-cluster integral $\beta$ defined by

$$
\beta=4 \pi \int_{0}^{\infty}\left[1-e^{-u(R) / k_{B} T}\right] R^{2} d R,
$$

with $k_{B}$ the Boltzmann constant and $T$ the absolute temperature. The quantity $\beta$ so defined in the perturbation theory represents the effective volume excluded to one segment by the presence of another, ${ }^{1}$ which, strictly speaking, might be distinguished from an analog defined in the polymer renormalization group theory. Since $u(R)$ is in general short ranged for nonionic polymers, the approximation is considered to be valid and has actually been working well as far as nonionic flexible polymers are concerned. In this paper, we consider the question of whether or not the approximation is still valid when the polymer chain becomes stiff. We note that such a question is, of course, outside the scope of the polymer renormalization group theory, which completely discards information about local structures of polymer chains.

For the present purpose, we carry out a Monte Carlo (MC) simulation study of the mean-square radius of gyration $\left\langle S^{2}\right\rangle$ and second virial coefficient $A_{2}$ for the freely rotating chains ${ }^{1,5}$ of bond angle $\theta$ ranging from $109^{\circ}$ to $175^{\circ}$ and with

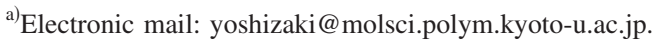

the Lennard-Jones (LJ) 6-12 potential $^{6}$ or the hard-sphere (HS) one. The chain of $\theta=109^{\circ}$ (approximately equal to the tetrahedral bond angle) is usually used as a model for flexible polymer chains, ${ }^{7-9}$ and the stiffness of the chain increases with increasing $\theta$ from $109^{\circ}$ to $175^{\circ}$. Considering the fact that the binary-cluster approximation works well for flexible chains, it is expected that we can find an appropriate diameter of the HS model so that both its $\left\langle S^{2}\right\rangle$ and $A_{2}$ agree with the corresponding ones of the LJ model with given energy parameters. In anticipation of results, we note that such a diameter really exists. Then the above question becomes more concrete, i.e., how the values of $\left\langle S^{2}\right\rangle$ and $A_{2}$ for the two interaction potentials come to be as $\theta$ is increased from $109^{\circ}$ to $175^{\circ}$.

\section{MODELS AND METHODS}

The model chain and the simulation algorithm used in this study are the same as those in the previous studies ${ }^{7,8}$ of $\left\langle S^{2}\right\rangle$ and $A_{2}$, except for the inclusion of the HS potential. Here we give brief descriptions of the model and algorithm.

The model chain is the freely rotating chain ${ }^{1,5}$ composed of $n$ bonds, each of length unity and of $n+1$ identical beads, whose centers are located at the $n-1$ junctions of two successive bonds and at the two terminal ends. The beads are numbered $0,1,2, \ldots, n$ from one end to the other, and the $i$ th bond vector connects the centers of the $(i-1)$ th and $i$ th beads with its direction from the $(i-1)$ th to the $i$ th bead. All the $n-1$ bond angles (not supplements) are fixed at $\theta$, so that the configuration of the entire chain may be specified by the set of $n-2$ internal rotation angles $\left\{\phi_{n-2}\right\}=\left(\phi_{2}, \phi_{3}, \ldots, \phi_{n-1}\right)$ apart from its position and orientation in an external Cartesian coordinate system, where $\phi_{i}$ is the internal rotation angle around the $i$ th bond vector.

The total intramolecular (excluded-volume) potential energy $U$ of the chain as a function of $\left\{\phi_{n-2}\right\}$ may be given by 


$$
U\left(\left\{\phi_{n-2}\right\}\right)=\sum_{i=0}^{n-4} \sum_{j=i+4}^{n} u\left(R_{i j}\right),
$$

where $u\left(R_{i j}\right)$ is the pair potential as a function of the distance $R_{i j}$ between the centers of the $i$ th and $j$ th beads. We must note here that the pairwise decomposability of the potential energy has been assumed, as is usually done in the field. ${ }^{1} \mathrm{We}$ also note that in Eq. (2) the interactions between the thirdneighbor beads along the chain have been neglected since they seem to make the chain locally take the cis conformation to excess in the case of the LJ 6-12 potential with an attractive interaction.

Similarly, the total intermolecular (excluded-volume) potential energy $U_{12}(1,2)$ between two chains 1 and 2 as a function of all the coordinates of chains 1 and 2 may be given by

$$
U_{12}(1,2)=\sum_{i_{1}=0}^{n} \sum_{i_{2}=0}^{n} u\left(R_{i_{1} i_{2}}\right),
$$

where $R_{i_{1} i_{2}}$ is the distance between the centers of the $i_{1}$ th bead of chain 1 and of the $i_{2}$ th one of chain 2 . We note that we use the McMillan-Mayer symbolism ${ }^{1,10}$ to formulate $A_{2}$ here and hereafter. Then the $i$ th bead $(i=0,1,2, \ldots, n)$ of chain $\alpha(\alpha=1,2)$ is labeled as $i_{\alpha}$, and the symbol $(\alpha)(\alpha$ $=1,2)$ denotes all the coordinates (external and internal) of chain $\alpha$.

As mentioned in Sec. I, we adopt as the pair potentials $u(R)$ the LJ 6-12 potential in a cutoff version and the HS one, the former being given by

$$
u(R)= \begin{cases}\infty & \text { for } 0 \leq R<c \sigma \\ u^{\mathrm{LJ}}(R) & \text { for } c \sigma \leq R<3 \sigma \\ 0 & \text { for } 3 \sigma \leq R,\end{cases}
$$

where $u^{\mathrm{LJ}}(R)$ is the LJ 6-12 potential given by ${ }^{6}$

$$
u^{\mathrm{LJ}}(R)=4 \epsilon\left[\left(\frac{\sigma}{R}\right)^{12}-\left(\frac{\sigma}{R}\right)^{6}\right],
$$

with $\sigma$ and $\epsilon$ the collision diameter and the depth of the potential well at the minimum of $u^{\mathrm{LJ}}(R)$, respectively. We note that $u(R)$ given by Eq. (4) is the LJ potential cutoff at the upper bound $3 \sigma$. The lower bound $c \sigma$ in Eq. (4) has been introduced for numerical convenience; the factor $c$ is properly chosen so that the Boltzmann factor $e^{-u^{\mathrm{LJ}}(c \sigma) / k_{B} T}$ may be regarded as numerically vanishing compared to unity. In practice, in double-precision numerical computation, we set

$$
c=\left[2 /\left(1+\sqrt{1+36 T^{*}}\right)\right]^{1 / 6},
$$

so that $e^{-u^{\mathrm{LJ} / k_{B} T}} \leq 2 \times 10^{-16}$ for $0 \leq R<c \sigma$, where $T^{*}$ is the reduced temperature defined by $T^{*}=k_{B} T / \epsilon$. On the other hand, the HS potential is simply written in the form ${ }^{6}$

$$
u(R)= \begin{cases}\infty & \text { for } 0 \leq R<\sigma_{c} \\ 0 & \text { for } \sigma_{c} \leq R,\end{cases}
$$

where $\sigma_{c}$ is the collision diameter.

The mean-square radius of gyration $\left\langle S^{2}\right\rangle$, i.e., the ensemble average of the square radius of gyration $S^{2}$, may be evaluated from

$$
\left\langle S^{2}\right\rangle=N_{s}^{-1} \sum_{\left\{\phi_{n-2}\right\}} S^{2}\left(\left\{\phi_{n-2}\right\}\right),
$$

where the sum is taken over $N_{s}$ sample configurations $\left\{\phi_{n-2}\right\}$ generated in a MC run by application of the pivot algorithm ${ }^{11,12}$ and the Metropolis method of importance sampling, ${ }^{13}$ as done in the previous MC study ${ }^{7}$ of $\left\langle S^{2}\right\rangle$. For each sample configuration, $S^{2}$ as a function of $\left\{\phi_{n-2}\right\}$ may be calculated from

$$
S^{2}=\frac{1}{n+1} \sum_{i=0}^{n}\left|\mathbf{r}_{i}-\mathbf{r}_{\text {c.m. }}\right|^{2},
$$

with $\mathbf{r}_{i}$ the vector position of the center of the $i$ th bead and $\mathbf{r}_{\mathrm{c} . \mathrm{m} \text {. }}$ the vector position of the center of mass of the chain given by

$$
\mathbf{r}_{\mathrm{c} . \mathrm{m} .}=\frac{1}{n+1} \sum_{i=0}^{n} \mathbf{r}_{i}
$$

In every $\mathrm{MC}$ run, an initial configuration is generated by trial and error so that all the distances between the centers of beads are greater than or equal to $c \sigma$ in the case of the $\mathrm{LJ}$ potential or $\sigma_{c}$ in the case of the HS one. One configuration is sampled at every $M_{\text {nom }}$ (nominal) pivot steps, so that $N_{s}$ $\times M_{\text {nom }}$ pivot steps are required to obtain a set of $N_{s}$ sample configurations.

As for the second virial coefficient $A_{2}$, it may be evaluated from ${ }^{8}$

$$
A_{2}=\frac{2 \pi N_{A}}{M^{2}} \int_{0}^{\infty}\left\{1-\exp \left[-\frac{\bar{U}_{12}(r)}{k_{B} T}\right]\right\} r^{2} d r,
$$

where $N_{A}$ is the Avogadro constant, $M$ is the molecular weight, and $\bar{U}_{12}(r)$ is the averaged intermolecular potential as a function of the distance $r=|\mathbf{r}|$ between the centers of mass of chains 1 and 2 defined by

$$
\bar{U}_{12}(r)=-k_{B} T \ln \left\langle\exp \left[-\frac{U_{12}(1,2)}{k_{B} T}\right]\right\rangle_{r} .
$$

In Eq. (12), $\langle\cdots\rangle_{r}$ indicates the conditional equilibrium average taken over the configurations of the two chains with $\mathbf{r}$ fixed by the use of the single-chain distribution function for each with the intramolecular excluded-volume potential given by Eq. (2). In practice, the conditional average may be calculated by the use of a set of $N_{s}$ sample configurations generated above, as follows. First, we randomly sample a pair of sample configurations (chains 1 and 2) from the set and calculate the intermolecular potential $U_{12}$ from Eq. (3) at given $r$ after randomizing the orientations of the two configurations in the external coordinate system. Numerical evaluation of $U_{12}$ may be carried out following the procedure used in the previous study of $A_{2}$ with the use of the "zippering" method. ${ }^{14,15}$ Then the average on the right-hand side of Eq. (12) may be evaluated from 


$$
\left\langle\exp \left[-\frac{U_{12}(1,2)}{k_{B} T}\right]\right\rangle_{r}=N_{p}^{-1} \sum_{(1,2)} \exp \left[-\frac{U_{12}(1,2)}{k_{B} T}\right],
$$

where $\Sigma_{(1,2)}^{\prime}$ indicates summation over $N_{p}$ pairs of sample configurations $(1,2)$ at a given $r$. With the values of $\bar{U}_{12}(r)$ so evaluated for various values of $r$ in the case of the LJ potential at a given $T^{*}$ or of the HS potential with a given $\sigma_{c}$, the quantity $A_{2} M^{2}$ for a given $n$ may then be calculated from Eq. (11) by numerical integration with the use of the trapezoidal rule formula.

In the practical evaluation of $\left\langle S^{2}\right\rangle$, we have generated ten sets of $10^{5}\left(=N_{s}\right)$ sample configurations for $n=6,10,20,50$, 100 , and 200, five sets of those for $n=500$, and two sets of those for $n=1000$. In each case, $M_{\text {nom }}$ has been chosen to be approximately $2 n$. In the evaluation of $\bar{U}_{12}$ (and $A_{2}$ ), $10^{6}$ or $10^{7}\left(=N_{p}\right)$ sample pairs have been taken from each set. Then the total number of sample pairs is equal to the number $N_{p}$ of sample pairs in each set multiplied by the number of sets. In the evaluations of both $\left\langle S^{2}\right\rangle$ and $A_{2}$, the parameter $\sigma$ in the LJ potential is set equal to unity.

All the numerical work has been done by the use of a personal computer with an AMD Athlon XP 2200+ CPU. A source program coded in C has been compiled by the GNU C compiler version 2.95 .4 with real variables of double precision. In the program, the subroutine package MT19937 supplied by Matsumoto and Nishimura ${ }^{16}$ has been used instead of the subroutine RAND included in the standard C library.

\section{RESULTS AND DISCUSSION}

\section{A. Mean-square radius of gyration}

In the second column of Table I are given the MC values of $\left\langle S^{2}\right\rangle / n$ for the freely rotating chains with the LJ potential at $T^{*}=8.0$ (good-solvent condition ${ }^{7}$ ) and with $\theta=109^{\circ}, 120^{\circ}$, $135^{\circ}, 165^{\circ}$, and $175^{\circ}$ and $n=6,10,20,50,100,200,500$, and 1000 , the number enclosed in parentheses attached to each value indicating its statistical error. The value and error for each chain are the mean and the standard deviation, respectively, of independent MC results. We note that the values for the chains with $\theta=109^{\circ}$ have been reproduced from Table I of Ref. 7 and Table III of Ref. 8

Figure 1 shows double-logarithmic plots of $\left\langle S^{2}\right\rangle / n$ against $n$. The open symbols represent the above-mentioned MC values for the chains with the LJ potential for $\theta=109^{\circ}$ $(\bigcirc), 120^{\circ}(\square), 135^{\circ}(\diamond), 165^{\circ}(\triangle)$, and $175^{\circ}(\nabla)$. The solid curves represent the theoretical values of $\left\langle S^{2}\right\rangle / n$ for the ideal freely rotating chain without interactions between beads, which have been calculated from

$$
\begin{aligned}
\left\langle S^{2}\right\rangle= & \frac{1}{6} \frac{1-\cos \theta}{1+\cos \theta} n+\frac{1}{6} \frac{1+6 \cos \theta-\cos ^{2} \theta}{(1+\cos \theta)^{2}} \\
& +\frac{1-1-7 \cos \theta+7 \cos ^{2} \theta+\cos ^{3} \theta}{6} \frac{1}{n+1} \\
& -\frac{2 \cos ^{2} \theta}{(1+\cos \theta)^{3}} \frac{1-(-\cos \theta)^{n+1}}{(n+1)^{2}},
\end{aligned}
$$

\begin{tabular}{|c|c|c|c|c|}
\hline \multirow[b]{2}{*}{$n$} & \multicolumn{2}{|c|}{$\left\langle S^{2}\right\rangle / n($ error $\%)$} & \multicolumn{2}{|c|}{$10 A_{2} M_{b}^{2} / N_{A}($ error $\%)$} \\
\hline & $\mathrm{LJ}$ & HS & $\mathrm{LJ}$ & HS \\
\hline \multicolumn{5}{|c|}{$\theta=109^{\circ}$} \\
\hline 6 & $0.289_{2}(0.1)$ & $0.288_{6}(0.1)$ & $5.12_{2}(0.2)$ & $4.98_{5}(0.1)$ \\
\hline 10 & $0.315_{8}(0.1)$ & $0.313_{4}(0.1)$ & $3.94_{2}(0.1)$ & $3.99_{1}(0.2)$ \\
\hline 20 & $0.359_{9}(0.1)$ & $0.354_{9}(0.1)$ & $2.98_{8}(0.1)$ & $3.10_{8}(0.1)$ \\
\hline 50 & $0.428_{7}(0.1)$ & $0.421_{9}(0.1)$ & $2.24_{5}(0.1)$ & $2.36_{3}(0.1)$ \\
\hline 100 & $0.486_{7}(0.1)$ & $0.480_{7}(0.1)$ & $1.86_{6}(0.1)$ & $1.96_{0}(0.1)$ \\
\hline 200 & $0.551_{1}(0.2)$ & $0.546_{2}(0.1)$ & $1.56_{7}(0.1)$ & $1.64_{3}(0.1)$ \\
\hline 500 & $0.647_{2}(0.1)$ & $0.645_{8}(0.1)$ & $1.25_{8}(0.0)$ & $1.31_{0}(0.1)$ \\
\hline 1000 & $0.729_{4}(0.1)$ & $0.729_{8}(0.1)$ & $1.06_{6}(0.1)$ & $1.10_{4}(0.1)$ \\
\hline \multicolumn{5}{|c|}{$\theta=120^{\circ}$} \\
\hline 6 & $0.353_{9}(0.1)$ & $0.354_{4}(0.1)$ & $5.39_{4}(0.2)$ & $5.22_{8}(0.1)$ \\
\hline 10 & $0.398_{7}(0.1)$ & $0.399_{1}(0.1)$ & $4.28_{4}(0.2)$ & $4.30_{5}(0.1)$ \\
\hline 20 & $0.463_{1}(0.1)$ & $0.463_{4}(0.1)$ & $3.37_{6}(0.1)$ & $3.49_{1}(0.1)$ \\
\hline 50 & $0.549_{1}(0.1)$ & $0.549_{1}(0.1)$ & $2.64_{2}(0.2)$ & $2.76_{8}(0.1)$ \\
\hline 100 & $0.615_{1}(0.1)$ & $0.617_{0}(0.1)$ & $2.24_{5}(0.2)$ & $2.35_{9}(0.1)$ \\
\hline 200 & $0.686_{7}(0.1)$ & $0.691_{1}(0.2)$ & $1.92_{1}(0.1)$ & $2.01_{7}(0.1)$ \\
\hline 500 & $0.794_{7}(0.1)$ & $0.800_{5}(0.1)$ & $1.56_{8}(0.1)$ & $1.63_{7}(0.1)$ \\
\hline 1000 & $0.886_{8}(0.1)$ & $0.899_{4}(0.0)$ & $1.34_{0}(0.0)$ & $1.39_{7}(0.1)$ \\
\hline \multicolumn{5}{|c|}{$\theta=135^{\circ}$} \\
\hline 6 & $0.458_{8}(0.0)$ & $0.459_{0}(0.0)$ & $5.43_{6}(0.2)$ & $5.41_{2}(0.1)$ \\
\hline 10 & $0.562_{3}(0.0)$ & $0.562_{5}(0.0)$ & $4.43_{0}(0.2)$ & $4.59_{7}(0.2)$ \\
\hline 20 & $0.710_{7}(0.1)$ & $0.711_{8}(0.1)$ & $3.65_{7}(0.3)$ & $3.92_{0}(0.2)$ \\
\hline 50 & $0.880_{0}(0.1)$ & $0.883_{4}(0.2)$ & $3.06_{7}(0.2)$ & $3.34_{2}(0.2)$ \\
\hline 100 & $0.982_{0}(0.1)$ & $0.989_{8}(0.1)$ & $2.73_{6}(0.2)$ & $2.98_{8}(0.1)$ \\
\hline 200 & $1.07_{4}(0.2)$ & $1.08_{6}(0.1)$ & $2.45_{3}(0.2)$ & $2.66_{7}(0.1)$ \\
\hline 500 & $1.19_{8}(0.0)$ & $1.21_{8}(0.3)$ & $2.10_{1}(0.1)$ & $2.27_{5}(0.1)$ \\
\hline 1000 & $1.30_{4}(0.1)$ & $1.33_{3}(0.2)$ & $1.85_{2}(0.1)$ & $2.00_{0}(0.1)$ \\
\hline \multicolumn{5}{|c|}{$\theta=165^{\circ}$} \\
\hline 6 & $0.638_{1}(0.0)$ & $0.638_{1}(0.0)$ & $5.36_{2}(0.4)$ & $5.55_{5}(0.3)$ \\
\hline 10 & $0.930_{7}(0.0)$ & $0.930_{7}(0.0)$ & $4.40_{5}(0.4)$ & $4.81_{8}(0.2)$ \\
\hline 20 & $1.59_{8}(0.0)$ & $1.59_{8}(0.0)$ & $3.73_{6}(0.5)$ & $4.28_{7}(0.2)$ \\
\hline 50 & $3.16_{1}(0.0)$ & $3.16_{1}(0.0)$ & $3.36_{0}(0.5)$ & $3.97_{1}(0.3)$ \\
\hline 100 & $4.82_{9}(0.0)$ & $4.82_{8}(0.1)$ & $3.22_{4}(0.2)$ & $3.84_{8}(0.4)$ \\
\hline 200 & $6.53_{4}(0.1)$ & $6.53_{4}(0.1)$ & $3.14_{7}(0.2)$ & $3.78_{3}(0.2)$ \\
\hline 500 & $8.19_{4}(0.1)$ & $8.20_{4}(0.0)$ & $3.07_{8}(0.4)$ & $3.70_{1}(0.2)$ \\
\hline 1000 & $8.92_{4}(0.3)$ & $8.95_{2}(0.1)$ & $3.03_{2}(0.1)$ & $3.64_{4}(0.2)$ \\
\hline \multicolumn{5}{|c|}{$\theta=175^{\circ}$} \\
\hline 6 & $0.663_{4}(0.0)$ & $0.663_{4}(0.0)$ & $5.31_{9}(0.3)$ & $5.57_{0}(0.2)$ \\
\hline 10 & $0.991_{9}(0.0)$ & $0.991_{9}(0.0)$ & $4.35_{0}(0.4)$ & $4.83_{3}(0.4)$ \\
\hline 20 & $1.80_{5}(0.0)$ & $1.80_{4}(0.0)$ & $3.64_{9}(0.4)$ & $4.31_{2}(0.5)$ \\
\hline 50 & $4.17_{1}(0.0)$ & $4.17_{1}(0.0)$ & $3.24_{8}(1.1)$ & $4.02_{2}(0.3)$ \\
\hline 100 & $7.88_{5}(0.0)$ & $7.88_{5}(0.0)$ & $3.10_{7}(0.8)$ & $3.92_{8}(0.6)$ \\
\hline 200 & $14.5_{5}(0.0)$ & $14.5_{5}(0.0)$ & $3.02_{9}(1.0)$ & $3.85_{9}(0.5)$ \\
\hline 500 & $29.8_{2}(0.0)$ & $29.8_{3}(0.0)$ & $2.98_{6}(1.4)$ & $3.83_{6}(0.2)$ \\
\hline 1000 & $45.5_{2}(0.0)$ & $45.5_{3}(0.0)$ & $2.94_{8}(0.7)$ & $3.82_{2}(0.1)$ \\
\hline
\end{tabular}

TABLE I. Results of MC simulation.

with the indicated values of $\theta$. For the chain of $\theta=109^{\circ}$, which corresponds to a typical flexible chain, the MC data points deviate upward from the corresponding solid curve as $n$ is increased because of the intramolecular excludedvolume effect. For the chains of $\theta=120^{\circ}$ and $135^{\circ}$, the situations are the same as that in the case of $\theta=109^{\circ}$ but the deviation of the MC value from the ideal one becomes small as $\theta$ is increased. As for the chains of $\theta=165^{\circ}$ and $175^{\circ}$, which correspond to typical semiflexible or stiff chains, the $\mathrm{MC}$ values almost agree with the corresponding ideal ones over the whole range of $n$ examined. We note that the in- 


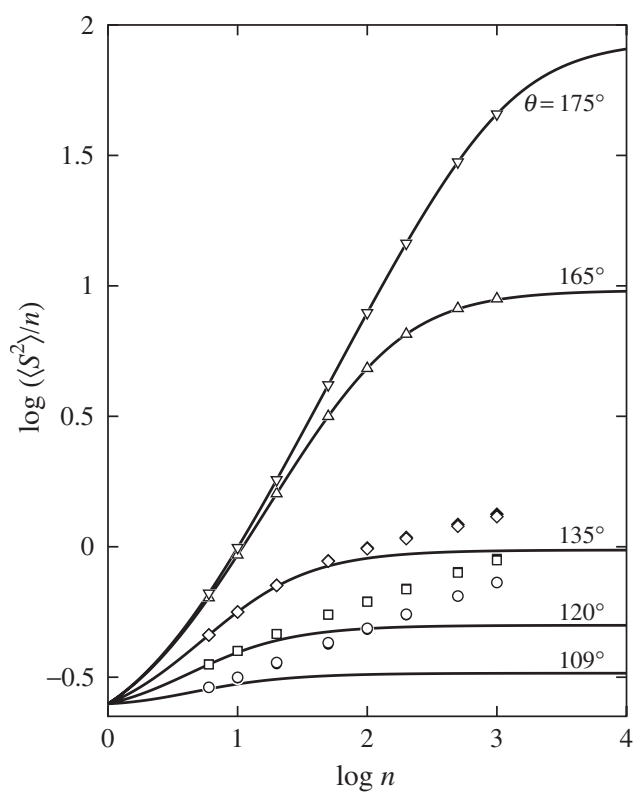

FIG. 1. Double-logarithmic plots of $\left\langle S^{2}\right\rangle / n$ against $n$. The open and closed symbols represent the MC values for the freely rotating chains with the LJ potential $T^{*}=8.0$ and the HS one with $\sigma_{c}=0.624$, respectively, for $\theta=109^{\circ}$ $(\bigcirc, \boldsymbol{\bullet}), 120^{\circ}(\square, \boldsymbol{\square}), 135^{\circ}(\diamond, \diamond), 165^{\circ}(\triangle, \boldsymbol{\Delta})$, and $175^{\circ}(\nabla, \boldsymbol{\nabla})$. The solid curves represent the theoretical values for the ideal freely rotating chain without interactions between beads calculated from Eq. (14) with the indicated values of $\theta$

tramolecular excluded-volume effect may be neglected for typical semiflexible or stiff chains of short chain lengths.

In the third column of Table I are also given the MC values of $\left\langle S^{2}\right\rangle / n$ for the freely rotating chains with the HS potential with $\sigma_{c}=0.624$, the $\sigma_{c}$ value being chosen so that the $\left\langle S^{2}\right\rangle / n$ values for the chains of $\theta=109^{\circ}$ are as close as possible to the corresponding values given in the second column for the chains with the LJ potential at $T^{*}=8.0$. It is seen that the values of $\left\langle S^{2}\right\rangle / n$ for the two freely rotating chains of each $\theta$ and $n$ agree with each other within approximately $2 \%$. In Fig. 1 are also shown the HS values by the closed symbols, although they are almost hidden by the open symbols (LJ values). We note that the agreement between the $\left\langle S^{2}\right\rangle / n$ values for the two chains of larger $\theta\left(165^{\circ}\right.$ and $\left.175^{\circ}\right)$ is rather natural since the intramolecular excluded-volume effect becomes immaterial for typical semiflexible or stiff chains with short chain lengths, as mentioned above. The results imply that we may assign a proper value to $\sigma_{c}$ of the chain with the HS potential so that its $\left\langle S^{2}\right\rangle$ agree well with that for the chain with the LJ potential at a given $T^{*}$ irrespective of the chain stiffness.

It is pertinent to make here a remark on the unperturbed value $\left\langle S^{2}\right\rangle_{0}$ of $\left\langle S^{2}\right\rangle$ without the excluded-volume effect. In a real polymer-solvent system, $\left\langle S^{2}\right\rangle_{0}$ means the $\left\langle S^{2}\right\rangle$ value in the $\Theta$ state which may be realized when repulsive and attractive interactions between repeat units (beads) cancel out each other but does not correspond to that of the ideal chain without any interactions between them. For the freely rotating chain with $\theta=109^{\circ}$, the $\Theta$ state may be reproduced by the use of the LJ potential at $T^{*}=3.72$ (and with $\sigma=1$ ) where $\left\langle S^{2}\right\rangle / n$ becomes a constant independent of $n$ for very large $n,^{7}$ which is approximately $20 \%$ larger than the asymptotic

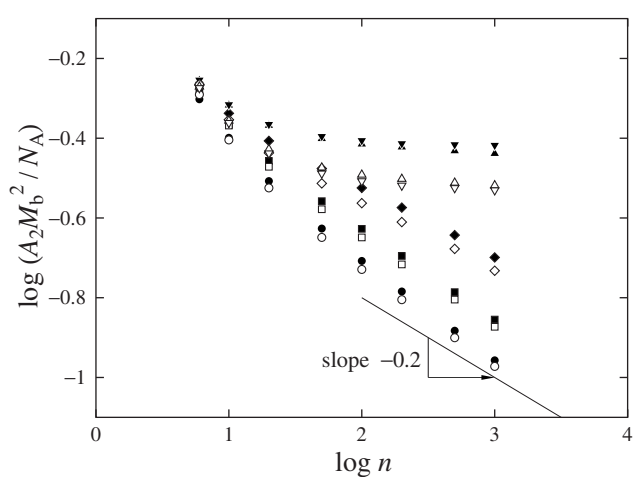

FIG. 2. Double-logarithmic plots of $A_{2} M_{b}^{2} / N_{A}$ against $n$. All the symbols have the same meaning as those in Fig. 1.

value (approximately 1/3) of $\left\langle S^{2}\right\rangle / n$ calculated from Eq. (14) for the ideal chain in the limit of $n \rightarrow \infty$. The implication is that the interactions between beads may appreciably affect $\left\langle S^{2}\right\rangle_{0}$ through the short-range interference. ${ }^{7,9,17}$ The situation is somewhat different in the case of the HS potential only with a repulsive core, by the use of which we cannot reproduce the real unperturbed chain but only the ideal one with $\sigma_{c}=0$. (Note that the chain with the LJ potential becomes the ideal one when $\sigma=0$.) Although the interaction between beads affects $\left\langle S^{2}\right\rangle_{0}$ through the short-range interference also in the case of the HS potential, we cannot evaluate it separately from $\left\langle S^{2}\right\rangle$ with the excluded-volume effect.

\section{B. Second virial coefficient and interpenetration function}

Now the problem is whether or not the values of $A_{2}$ for the two freely rotating chains considered above agree with each other irrespective of the chain stiffness. In the fourth and fifth columns of Table I are given the MC values of $A_{2} M_{b}^{2} / N_{A}$ for the chains with the LJ potential at $T^{*}=8.0$ and with the HS one with $\sigma_{c}=0.624$, respectively, where $M_{b}$ $(=M / n)$ is the molecular weight per bond, the number enclosed in parentheses attached to each value indicating its statistical error.

Figure 2 shows double-logarithmic plots of $A_{2} M_{b}^{2} / N_{A}$ against $n$, all the symbols having the same meaning as those in Fig. 1. In the case of $\theta=109^{\circ}$, which corresponds to a typical flexible chain, the values of $A_{2}$ for the chain with the LJ potential $(\bigcirc)$ are slightly (5\% at most) smaller than the corresponding ones for the chain with the HS potential ( $)$ for all $n$ except $n=6$. For both the chains, $A_{2}$ decreases with increasing $n$ and the slopes of the plots become almost identical with the asymptotic value of -0.2 for very large $n$. For a given $n, A_{2}$ increases with increasing $\theta$ in the range of large $n$. In the case of $\theta=175^{\circ}$, which corresponds to a typical semiflexible or stiff chain, $A_{2}$ becomes almost independent of $n$ for $n \geq 100$, as in the case of the rigid rod.

It is more important to see in Fig. 2 that as $\theta$ (chain stiffness) is increased, the value of $A_{2}$ for the chain with the $\mathrm{LJ}$ potential becomes remarkably smaller than the corresponding value for the chain with the HS one and that $A_{2}$ for the former chain with a given $n$ has a maximum at $\theta=165^{\circ}$, while it increases monotonically with increasing $\theta$ for the 


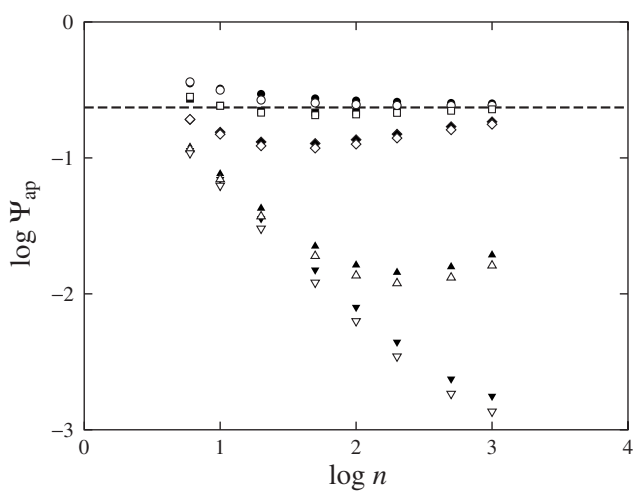

FIG. 3. Double-logarithmic plots of $\Psi_{\text {ap }}$ against $n$. All the symbols have the same meaning as those in Fig. 1. The dashed horizontal line represents the asymptotic value of 0.235 of $\Psi_{\text {ap }}$ obtained by Barrett (Ref. 22).

latter. It may be regarded as arising from the fact that two chains with the LJ potential (having the attractive interaction) which are close to each other prefer to be parallel rather than perpendicular to each other when their stiffness becomes large and their $A_{2}$ may therefore be suppressed, as discussed by van der Schoot and Odijk ${ }^{18}$ in the case of long rigid rods with a van der Waals-type potential.

Finally, we examine the behavior of the apparent interpenetration function $\Psi_{\text {ap }}$ defined by ${ }^{19}$

$$
A_{2}=4 \pi^{3 / 2} N_{A} \frac{\left\langle S^{2}\right\rangle^{3 / 2}}{M^{2}} \Psi_{\text {ap }}
$$

from the whole $A_{2}$ including the effects of chain ends ${ }^{20,21}$ as a function of $n$. Figure 3 shows double-logarithmic plots of $\Psi_{\text {ap }}$ against $n$, where the values of $\Psi_{\text {ap }}$ have been calculated from Eq. (15) with the MC values of $\left\langle S^{2}\right\rangle / n$ and $A_{2} M_{b}^{2} / N_{A}$ given in Table I. All the symbols have the same meaning as those in Fig. 1 and the dashed horizontal line represents the asymptotic value of 0.235 of $\Psi_{\text {ap }}$ (or $\Psi$ without the effects of chain ends) obtained by Barrett. ${ }^{22}$

In the case of $\theta=109^{\circ}$, the values of $\Psi_{\text {ap }}$ for the chains with the LJ and HS potentials agree with each other within $7 \%$ at most, which is a natural consequence of the results for $\left\langle S^{2}\right\rangle$ and $A_{2}$ for the two chains shown in Figs. 1 and 2, respectively, and they decrease monotonically with increasing $n$ and seem to approach the above asymptotic value. In the cases of $\theta=120^{\circ}, 135^{\circ}$, and $165^{\circ}, \Psi_{\text {ap }}$ for the chains with a given $n$ decreases with increasing $\theta$ and $\Psi_{\text {ap }}$ as a function of $n$ exhibits a minimum and increases monotonically with increasing $n$ after passing through the minimum. In the case of $\theta=175^{\circ}, \Psi_{\text {ap }}$ does not exhibit a minimum and decreases monotonically with increasing $n$ in the whole range of $n$ examined. We note that even in this case, $\Psi_{\text {ap }}$ approaches the asymptotic value in the limit of $n \rightarrow \infty$ after passing through a minimum at some value of $n$ larger than 1000 . It is seen that the larger downward deviation from the asymptotic value is, the larger difference between the values of the two chains with the LJ and HS potentials is.

From the MC results shown in Figs. 2 and 3, it may be concluded that the intermolecular excluded volume of the chain with the LJ potential becomes smaller than that of the corresponding chain with the HS one as the chain stiffness is increased, even if $\sigma_{c}$ is properly determined so that the intramolecular excluded volumes for the two chains agree well with each other. In other words, the binary-cluster approximation does not seem to work well for typical semiflexible and stiff polymers.

\section{CONCLUSION}

We have examined the behavior of the mean-square radius of gyration $\left\langle S^{2}\right\rangle$ and second virial coefficient $A_{2}$ for two freely rotating chains with the LJ 6-12 and HS potentials under a good-solvent condition by MC simulation, giving major attention to the effect of chain stiffness on the intraand intermolecular excluded volumes. It is shown that we may satisfactorily assign the value of 0.624 to the collision diameter $\sigma_{c}$ of the HS potential so that $\left\langle S^{2}\right\rangle$ of the chain with the HS potential agrees well with that of the chain with the $\mathrm{LJ}$ one at the reduced temperature $T^{*}=8.0$ irrespective of the chain stiffness (i.e., the bond angle $\theta$ of the freely rotating chain in the range from $109^{\circ}$ to $175^{\circ}$ ). It is then found that $A_{2}$ of the latter chain becomes remarkably smaller than that of the former as the chain stiffness $(\theta)$ is increased, in contrast to the case of $\left\langle S^{2}\right\rangle$. The result implies that the binarycluster approximation does not seem to work well for typical semiflexible and stiff polymers.

${ }^{1}$ H. Yamakawa, Modern Theory of Polymer Solutions (Harper \& Row, New York, 1971); Its electronic edition is available on-line at http:// www.molsci.polym.kyoto-u.ac.jp/archives/redbook.pdf

${ }^{2}$ P.-G. de Gennes, Scaling Concepts in Polymer Physics (Cornell University, Ithaca, NY, 1979).

${ }^{3}$ K. F. Freed, Renormalization Group Theory of Macromolecules (Wiley, New York, 1987).

${ }^{4}$ J. des Cloizeaux and G. Jannink, Polymers in Solution, Their Modelling and Structure (Clarendon, Oxford, 1990).

${ }^{5}$ P. J. Flory, Statistical Mechanics of Chain Molecules (Interscience, New York, 1969).

${ }^{6}$ J. P. Hansen and I. R. McDonald, Theory of Simple Liquids, 3rd ed. (Academic, London, 2006).

${ }^{7}$ H. Yamakawa and T. Yoshizaki, J. Chem. Phys. 118, 2911 (2003).

${ }^{8}$ H. Yamakawa and T. Yoshizaki, J. Chem. Phys. 119, 1257 (2003).

${ }^{9}$ H. Yamakawa and T. Yoshizaki, J. Chem. Phys. 121, 3295 (2004).

${ }^{10}$ W. G. McMillan and J. E. Mayer, J. Chem. Phys. 13, 276 (1945).

${ }^{11}$ M. Lal, Mol. Phys. 17, 57 (1969).

${ }^{12}$ N. Madras and A. D. Sokal, J. Stat. Phys. 50, 109 (1988).

${ }^{13}$ N. Metropolis, A. W. Rosenbluth, M. N. Rosenbluth, A. H. Teller, and E. Teller, J. Chem. Phys. 21, 1087 (1953).

${ }^{14}$ S. D. Stellman and P. J. Gans, Macromolecules 5, 516 (1972).

${ }^{15}$ S. D. Stellman, M. Froimowitz, and P. J. Gans, J. Comput. Phys. 7, 178 (1971).

${ }^{16}$ M. Matsumoto and T. Nishimura, ACM Trans. Model. Comput. Simul. 8, 3 (1998); The subroutine package MT19937 is available online at http:// www.math.sci.hiroshima-u.uc.jp/ m-mat/MT/emt.html.

${ }^{17}$ W. Bruns, Macromolecules 17, 2826 (1984).

${ }^{18}$ P. van der Schoot and T. Odijk, J. Chem. Phys. 97, 515 (1992).

${ }^{19}$ Y. Einaga, F. Abe, and H. Yamakawa, Macromolecules 26, 6243 (1993).

${ }^{20}$ H. Yamakawa, Macromolecules 25, 1912 (1992).

${ }^{21}$ H. Yamakawa, Helical Wormlike Chains in Polymer Solutions (Springer, Berlin, 1997).

${ }^{22}$ A. J. Barrett, Macromolecules 18, 196 (1985). 